\title{
Nephrotic syndrome due to membranous nephropathy as the cause of rising TSH levels or primary hypothyroidism as the cause of nephrotic syndrome?: A case report
}

\author{
Sohini Patel ${ }^{i}$, Dr. Nirav Gandhi ii and Dr. Jayadave Shakher iii \\ Please send correspondence to: Dr. Jayadave Shakher, jayadave.shakher@heartofengland.nhs.uk
}

\section{Introduction}

Primary hypothyroidism is the most common form of hypothyroidism in the UK: it has a prevalence of $3-8 \%$ worldwide ${ }^{1}$.

Its disruption to the pituitary-thyroid axis results in decreased levels of thyroxine (T4) and triiodothyronine (T3) being available to the body. Consequential increases in thyroid stimulating hormone (TSH) are thereafter compensatory. The management in primary hypothyroidism is therefore to replace the shortfall with levorotatory forms of thyroxine (T4) ${ }^{2}$.

Nephrotic syndrome is a triad of proteinuria $(>3 \mathrm{~g} / 24 \mathrm{~h}$ or a spot urine protein:creatinine ratio of $>300-350 \mathrm{mg} /$ mmol), hypoalbunaemia (albumin $<25 \mathrm{~g} / \mathrm{L}$ ), and oedema ${ }^{3}$. It is usually also associated with severe hyperlipidaemia (total cholesterol $>10 \mathrm{mmol} / \mathrm{L}$ ), however this can also be seen in patients with hypothyroidism ${ }^{4}$

Causes of nephrotic syndrome are numerous, but are crudely divided into primary and secondary. Primary causes are those in which the kidney itself is subject to injury such as glomerulonephritis. However secondary causes are much more common.

Although many causes of rising TSH are documented, one unusual cause to consider is nephrotic syndrome, whereby the permeability of the kidneys predisposes to a loss of proteins (proteinuria) including thyroid-binding globulin. This, alongside losses of the protein-based thyroid hormones, means that TSH rises to attempt compensation (figure 2).

This case looks at an example of rising TSH levels despite increasing therapeutic thyroxine and the consequential investigations into the cause.

\section{Case}

A 57 year old gentleman with known hypothyroidism, managed on 200 micrograms thyroxine, presented with elevated thyroid stimulating hormone (TSH) levels. His hypothyroidism was previously well managed and the patient had been on treatment for almost 14 years with no issues of compliance.

In addition to the raised TSH, the patient also noted progressive leg swelling and some associated shortness of breath over the preceding 12 months. This was confirmed on examination with pitting oedema up to the abdomen with concomitant scrotal swelling. Furthermore, there was hypertension (175/103) with no postural drop noted. There was no indication of thyroid autoimmune complexes as there was iodine deficiency involvement. His initial blood results demonstrated a low albumin of 21 (previously 44) and his urinanalysis confirmed marked proteinuria $(+3)$ in addition to +2 blood. Therefore, nephrotic syndrome was investigated as a probable cause. Urine protein was raised at $12.42 \mathrm{~g} / \mathrm{L}(<0.15)$ as was RBC count. Blood results included a raised Beta-2microglobulin and the presence of ribonucleic protein (RNP). The kidney biopsy demonstrated membranous glomerulonephritis due to thickened basement membranes as a result of segmental proliferation.

This was symptomatically managed on frusemide $40 \mathrm{mg}$ $\mathrm{BD}$ and ramipril 10mg OM. Weight loss, functional renal improvement and a reduction in oedema resulted.

\section{Conclusions}

Identifying these biochemical changes and performing a simple urine dipstick would provide the adequate information to diagnose nephrotic syndrome ${ }^{21}$. Management of the increase in TSH will not be aided by serially increasing the thyroxine replacement, as the carrying capacity of the hormone will still remain diminished. Instead, one must manage the nephrotic syndrome. In broad terms this is by maintenance of euvolaemia and osmolality of the blood. Dietary alterations including low-salt and high-protein intake will improve TBG concentrations. Additionally, medical interventions such as diuretics will further maintain appropriate fluid balance and ACE-inhibitors can preserve appropriate blood pressure, which is also proved to decrease protein loss.

It is important to recognise that this is one axis in endocrinology and a great many hormones are protein based, as are their carrier molecules (such as albumin). The hypothalamic-pituitary and growth hormone axis may also warrant closer monitoring 22

In conclusion, this case emphasises that in the event of rising TSH, despite good compliance and no significant comorbidity or drug interaction, a simple urine dipstick could reveal an alternative causal mechanism that can then be subsequently investigated and managed appropriately. It also serves as a reminder that proteinuria can have far reaching repercussions in endocrinology. Although it may be difficult to determine the causal link between primary hypothyroidism and nephrotic syndrome we wish to encourage further evidence-based trials into this association, to provide a more substantial pool of data to analyse.

\section{Discussion}

Many causes are known for nephrotic syndrome, with membranous nephropathy accounting for $20-30 \%$ of all nephrotic syndrome causes, most recognisably, there is marked thickening of the glomerular basement membrane. Membranous nephropathy in turn has associated autoimmune components, thus contributing to a potential cause of unexplained proteinuria. Although there is an association between autoimmune components of thyroid disease and glomerulonephritis, no causal link has been proven ${ }^{6,7}$. Additionally, there is substantial evidence proving the association between primary hypothyroidism and congenital nephrotic syndrome, but similar causal correlations in adults have not yet been substantiated ${ }^{8-13}$. In our case this gentleman had no indication of any autoimmune involvement, and hence it may be reasonable to suggest that there is little indication of his hypothyroidism to have caused his symptomatic nephrotic syndrome. But this is difficult to determine and the reverse can be argued equally, this is demonstrated when looking at the importance of thyroid hormones.

Thyroid hormone activity cannot be underestimated as it is required for homeostasis, development and affects many organs in the body including the kidney ${ }^{14}$. As such, in patients with hypothyroidism and hence low levels of T4 and T3, renal function has been shown to decline through worsening creatinine levels and dropping GFR's. Therefore, there is significant room for a patient's hypothyroidism to lead to worsening of renal function and hence potentiate nephrotic syndrome ${ }^{14}$. This does not only go to show the role of thyroid hormones in renal function but the importance of appropriate medical management of thyroid hormones in hypothyroidism. Our patient was fully compliant with his levothyroxine over the 14 years of his thyroid disease, suggesting that the pathological mechanism described above may have contributed to the decline in renal function as opposed to a lack of compliance to his medication.

The means by which nephrotic syndrome can result in rising levels of TSH, are explained in principle by the levels of thyroxine-bindingglobulin (TBG). TBG is the core protein responsible for transportation of T4 and T3 in the bloodstream (albumin and transthyretin are also minor contributors in this task) ${ }^{15,16}$. As a protein, it is subject to excessive excretion in nephrotic syndrome, along with free T4 and T3 (protein hormones). Thus, resulting in diminished carrying capacity and therefore efficacy of these thyroid hormones- despite adequate supplementation. The feedback mechanism attempts to compensate this imbalance by increasing output of TSH from the pituitary gland ${ }^{15,17-20}$. The following diagram summarises the biochemical changes in a normal functioning axis compared to a skewed axis due to nephrotic syndrome (figure 2):

O

0

O

O

0

\section{Literature cited}
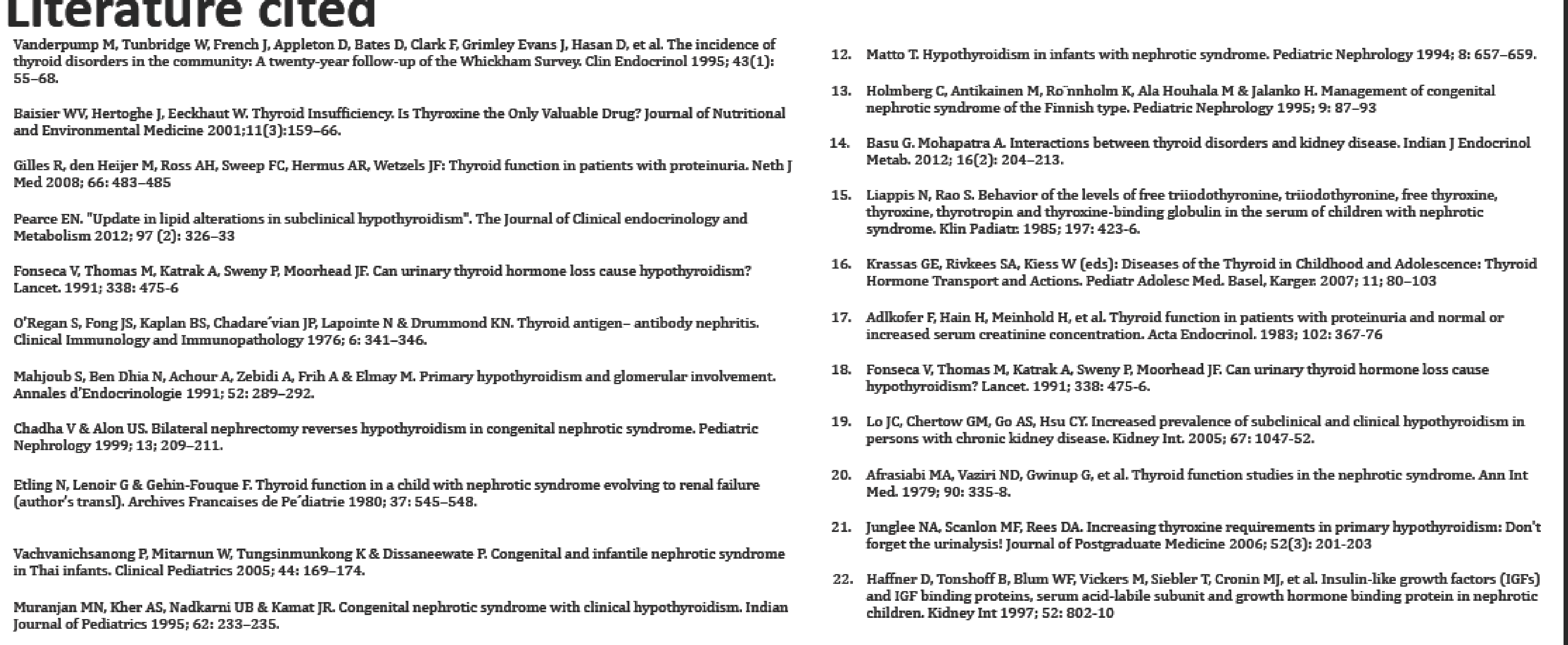

\section{Acknowledgments}

Thank you to Matt Jenkins iv, Jaskanwal Sara v, Karan Jolly vi, Tanveer Chohan vii for their contribution.

in

ii $B S c$ (Hon $)$ (2)
Trust, UK)

iiii Dip. Diab., M.D., MRCP, MBA (Consultant Physician in Diabetes and Endocrinology, Birmingham Heartlands Hospital, Heart of
England Foundation Trust, UK)

iv MBChB (Waikato General Hospital, Hamilton, New Zealand)

$\checkmark$ MBChB (University Hospital Coventry and Warwickshire, UK)

vi MBChB (Walsall Healthcare NHS Trust, UK)

vii MBChB (University Hospitals Birmingham NHS Foundation Trust, UK) 